Paper ID \#19736

\title{
Keeping Current: An Update on the Structure and Evaluation of a Program for Graduate Women Interested in Engineering Academia
}

\author{
Ms. Nicole D. Jackson, University of Illinois at Urbana-Champaign
}

Nicole D. Jackson is a third-year PhD student in the Civil and Environmental Engineering department at the University of Illinois at Urbana-Champaign (UIUC), and is a member of Megan Konar's group. Her research focuses on applying big data to understand the food-water nexus to promote food security. Also, she is currently a co-coordinator for the Illinois Female Engineers in Academia Training program as well as the Girls' Adventures in Mathematics, Engineering, and Science camp for environmental engineering and sustainability.

\section{Ms. Kaitlin I. Tyler, University of Illinois, Urbana-Champaign}

Kaitlin received her BS in Materials Science and Engineering with a concentration in biomaterials from Michigan State University in 2012. She is currently working on her PhD at the University of Illinois Urbana Champaign under Professor Paul Braun. Her research focuses on manipulating eutectic material microstructures for optical applications. She is also one of the co-coordinators for Girls Learning About Materials (GLAM), a summer camp for high school girls interested in engineering.

Mrs. Yanfen Li, University of Illinois, Urbana-Champaign

Yanfen Li is a Ph.D student in the Department of Bioengineering at the University of Illinois at UrbanaChampaign working under Dr. Kris Kilian. Her research focus is on biomaterials and tissue engineering.

Wan-Ting Chen, University of Illinois, Urbana Champaign

Wan-Ting Chen is a Ph.D. candidate in the Department of Agricultural and Biological Engineering at the University of Illinois at Urbana-Champaign, working under the guidance of Professor Yuanhui Zhang. Wan-Ting received her B.Sc in Chemical Engineering from the National Taiwan University and M.S. in Agricultural and Biological Engineering from the University of Illinois. Wan-Ting's research work has been in the context of developing a synergistically integrated waste-to-energy system, EnvironmentEnhancing Energy (E2-Energy), that simultaneously produces biofuel, treats wet biowaste and captures carbon dioxide via algae growing and hydrothermal liquefaction (HTL). Wan-Ting's ongoing work focuses on upgrading of the HTL biocrude oil converted from wet biowaste into transportation fuels by distillation, esterification, thermal cracking, and hydroprocessing with catalysts. Wan-Ting has been a SWE member since 2012 and is aiming for a future career in academia.

Chaoyang Liu, University of Illinois, Urbana-Champaign

Prof. Rohit Bhargava, University of Illinois, Urbana-Champaign

Rohit Bhargava is Founder Professor of Engineering at the University of Illinois at Urbana-Champaign with affiliations across several departments (Primary - Bioengineering: Affiliated - Electrical and Computer Engineering, Mechanical Science and Engineering, Chemical and Biomolecular Engineering and Chemistry) as well as the Beckman Institute for Advanced Science and Technology. Rohit received dual B.Tech. degrees (in Chemical Engineering and Polymer Science and Engineering) from the Indian Institute of Technology, New Delhi in 1996 and his doctoral thesis work at Case Western Reserve University (Department of Macromolecular Science and Engineering) was in the area of polymer spectroscopy. He then worked as a Research Fellow at the National Institutes of Health (2000-2005) in the area of biomedical vibrational spectroscopy. Rohit has been at Illinois since as Assistant Professor (2005-2011), Associate Professor (2011-2012) and Professor (2012-). Rohit was the first assistant professor hired into the new Bioengineering department and played a key role in the development of its curriculum and activities. He later founded and serves as the coordinator of the Cancer Community @ Illinois, which is slated to become the first technology-focused cancer center in the nation. Research in the Bhargava laboratories focuses on fundamental theory and simulation for vibrational spectroscopic imaging, developing new instrumentation 
and developing chemical imaging for molecular pathology. Using 3D printing and engineered tumor models, recent research seeks to elucidate hetero-cellular interactions in cancer progression. Rohit's work has been recognized with several research awards nationally. Among recent honors are the Agilent Thought Leader award (2016, election as Fellow of the AIMBE and SAS (2015), Meggers Award (Society for applied spectroscopy, 2014), Craver Award (Coblentz Society, 2013) and the FACSS Innovation Award (2012). Rohit has also been recognized for his dedication to teaching in the College of Engineering (Rose and Everitt awards) and he is routinely nominated to the list of teachers ranked excellent at Illinois. 


\title{
Keeping Current: An Update on the structure and evaluation of a program for graduate women interested in engineering academia
}

\begin{abstract}
According to data from ASEE, women were awarded $23.1 \%$ of doctoral degrees and held $15.7 \%$ of tenured/tenuretrack faculty faculty positions in 2015 versus $21.3 \%$ and $12.7 \%$ in 2009 , respectively [1,2]. While promising, the leaky pipeline remains a persistent problem in the recruitment of underrepresented people into tenure track positions. To help overcome this barrier, engineering graduate students at the University of Illinois at Urbana-Champaign created the Illinois Female Engineers in Academia Training (iFEAT) program to improve the competitiveness of underrepresented applicants in the tenure-track faculty recruitment process. The program is two-fold. First, seminars and panel discussions lead by faculty representing different engineering disciplines cover a variety of topics related to the job search process. Secondly, peer review sessions over the course of several months allow students to develop their own application materials. Since its founding in 2014, the program has been evaluated by considering four elements: content, format, pace, and climate. The evaluations in the first two years were based on conducting pre-, mid-, and post-surveys as well as voluntary one-on-one exit interviews. For the programs third year, we made significant changes based past participant feedback. Specific topics were expanded to increase understanding and improve familiarity with the application process. The evaluation structure was revised to increase the amount of immediate feedback. In this paper, we discuss how the program has evolved over the three years as well as how our methods for program monitoring have been revised. By incorporating these changes, we hope to continue to prepare high quality female faculty candidates, thereby diminishing the gender gap in engineering academia.
\end{abstract}

\section{Introduction}

The STEM fields have demonstrated a persistent gender gap at all education levels and faculty positions. This well-known gap has also lead to continuous discussion on how to address and overcome it [3-6]. Most recent data shows that women were awarded $23.1 \%$ of doctoral degrees and held $15.7 \%$ of tenured/tenuretrack faculty faculty positions in 2015 versus $21.3 \%$ and $12.7 \%$ in 2009 , respectively [1,2]. However, the data presents a complex yet slightly bleaker picture for ethnic minorities such as African-Americans and Hispanics over the same period. The percentage of PhDs awarded to African-Americans declined from $3.8 \%$ to $3.2 \%$, whereas the percentage of Hispanic PhDs increased nationally from $3.8 \%$ to $6.1 \%$. In 2015 , African-Americans and Hispanics comprise $2.5 \%$ and $3.9 \%$ of all tenured/tenure-track faculty positions, respectively [1]. The common theme across these groups is the noticeable decline in representation of these populations among faculty ranks.

Universities have begun to develop their own initiatives to address the lack of diversity amongst faculty. Broadly speaking, these initiatives can be grouped into two classifications: pipeline or climate-focused [7]. On our campus, we have created a program that addresses the pipeline problem by considering lack of preparedness and familiarity as the two dominant issues that can prohibit women in engineering from considering faculty positions upon graduation $[8,9]$.

Surveys have been consistently used throughout our program to track participant development at the initial, midpoint, and ends. In our first two years, our understanding of how participants felt in terms of knowledge gains as well as material preparedness was limited to these three surveys. When we began planning for our third year, we felt that we needed more data to better understand how participants were reacting to our programming, especially when taking into account the significant changes we were proposing. More specifically, our prior survey framework was limiting our understanding of whether or not we were truly helping 
participants improve their familiarity and preparedness to become competitive in the faculty job market. In other words, we wondered if individual sessions were beneficial to participants in terms of enhancing their preparation of faculty materials and contributed to their overall familiarity.

In this paper, we expand our program evaluation significantly in the third year by administering surveys at every session. This enables us to address the following questions: (1) How does increased surveying aid in understanding participant development? (2) How can survey data be incorporated to update programming? (3) How does faculty self-selection of session participation affect interaction with participants?

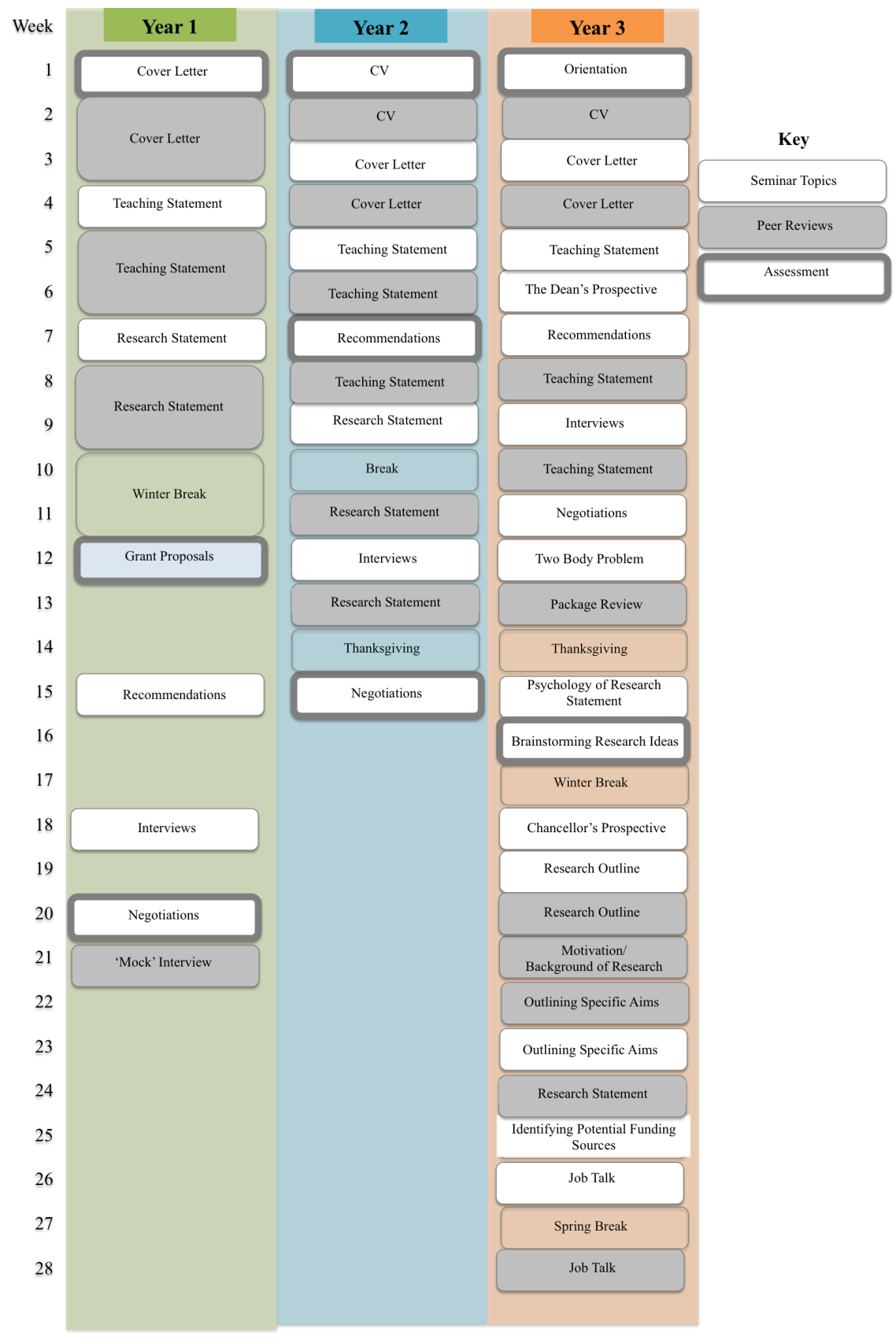

Figure 1: Comparison of iFEAT programming by year. Year 3 saw an increase in programming such that it encompassed nearly the duration of an entire Fall and Spring semester. 


\section{Prior program structure}

The Illinois Female Engineers in Academia Training (iFEAT) program began in Fall 2014 at the University of Illinois at Urbana-Champaign with eight female graduate students in engineering. The program was designed to cover the major components of the faculty job application with the tangible goal of each participant having a peer-reviewed application packet at the program's conclusion. The 2014-2015 program consisted of six seminar topics, four peer review sessions, and three assessments (see Figure 1). The program started shortly after the midpoint of the Fall semester and carried over for two sessions into the Spring semester. The cohort was equally divided into peer review groups. Participants were given approximately two weeks to self-arrange peer review sessions of their materials.

The first ever participants noted varying peer review experiences depending on the organizational level of their review group. Although participants were given two weeks to self-organize, we were told that it was difficult to find common meeting times to conduct a peer review. Thus, we decided to formally add the peer review to the program calendar and were treated as simply another session. Given the focus of our participants on searching for research positions (see Figure 2), the second program year saw the peer review opportunities for the research and teaching statements double.

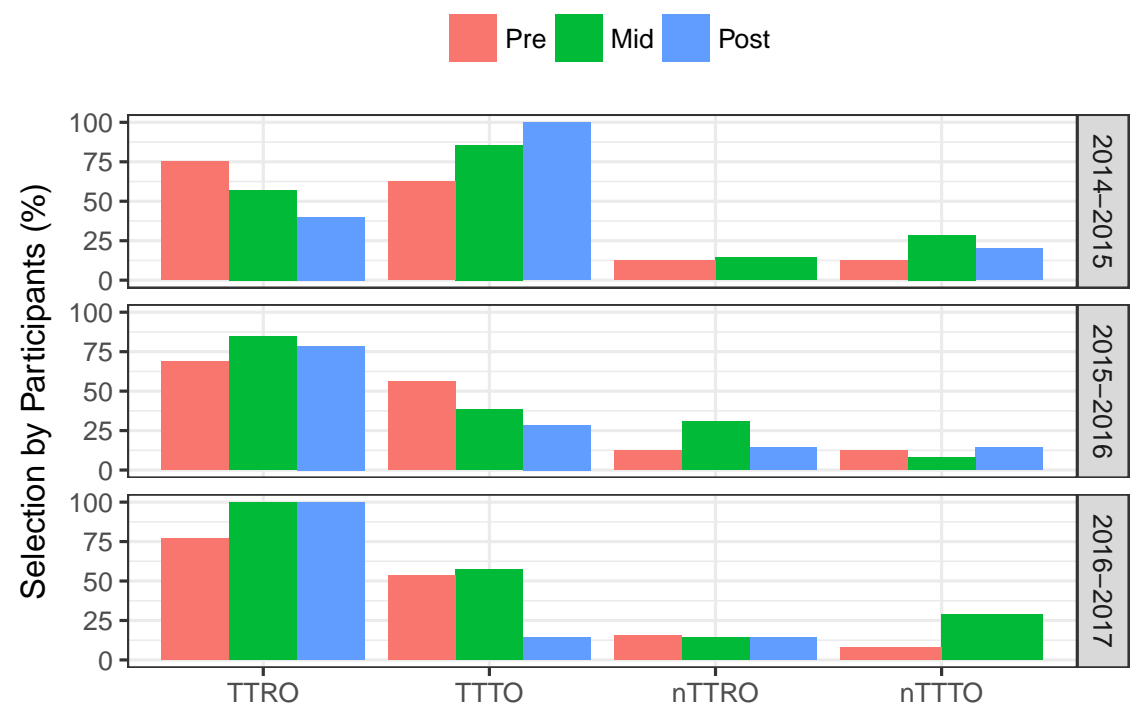

Figure 2: Selection of types of faculty positions by participant where TTRO refers to tenure-track, research oriented; TTTO refers to tenure-track, teaching oriented; nTTRO refers to non-tenure track, research oriented; and nTTTO refers to non-tenure track, teaching oriented. Tenure track-type positions are preferred by participants in all program years compared to non-tenure tack positions.

During the 2015-2016 program, we also observed a noticeable decline in attendance when we entered into the research statement part of the timeline (see Figure 10). The level of attrition was surprising given the consistent desire by participants to continue to conduct research in an academic setting. Participants later admitted that they were overwhelmed by the statement and had difficulty in developing research concepts that differentiated themselves from their advisors.

In order to facilitate evaluation of our program during the first two years, participants were voluntarily 
allowed to complete pre-, mid-, and post-surveys. These surveys were designed to help understand career plans (i.e., the types of institutions participants aspired to apply to after completing the program) as well as changes in confidence and preparedness over time $[8,9]$. At the conclusion of these years, participants were also offered the opportunity to share their experiences via interview.

\section{Methods}

\section{Participant surveys}

The 2016-2017 program saw a continuation of the pre-, mid-, and post- surveys that were hallmarks of the previous years. Likert scales were used to assess preparation (not at all prepared to very prepared) and familiarity (not at all familiar to very familiar) at each program interval. We asked participants to identify the types of institutions they were interested in pursuing a career at. Institutions choices were limited to the following categories: research intensive institutions ('R1'), research and teaching balanced ('R2'), teaching intensive institutions ('teaching'), and community colleges ('CC). They were were also asked what types of positions they were interested in. Position options were limited to the following: tenure-track, research oriented ('TTRO'); tenure-track, teaching oriented ('TTTO'); non tenure-track, research oriented ('nTTRO'); and non tenure-track, teaching oriented ('nTTTO').

For the current program year, we added a three question survey for each session to estimate how participant knowledge and preparation changed by attending panels/seminars and peer reviews, respectively. The first question on the survey was to be answered before the session began to obtain baseline data from the participants. The remaining two questions were answered at the conclusion of the session. For seminars and panels, participants were asked to rate their knowledge of the topic after the session and the knowledge level of the speakers. Similarly, participants were asked to evaluate the level of preparation and how helpful the feedback was at the conclusion of the peer review session. All short surveys had a space to add in written comments.

\section{Faculty surveys}

Campus faculty serve as speakers for the panels and seminars offered throughout the program. In Years 1 and 2, faculty were contacted approximately two weeks in advance to ask if they were available to partake in a session on a given topic. Although the requests were last minute, we were able to secure 15 and 16 faculty to contribute to the 2014-2015 and 2016-2017 programs, respectively. However, time conflicts were a serious issues such that four and five faculty from 2014-2015 and 2015-2016 program years, respectively, were unable to contribute to sessions.

We introduced a faculty interest survey (FIS) in the 2016-2017 program year to help lessen time conflicts for faculty and allow them the opportunity to select sessions that they were most interested in contributed to. The FIS were created separately for the Fall and Spring semesters, and were disseminated to 23 faculty in July /early August and and 13 faculty in November. They were given approximately two weeks respond. The difference in distribution levels results from the Fall semester having more seminars (10) than the Spring (5) (see Figure 1).

\section{Results and discussion}

\section{Participant professional interests and preparedness}

Figure 2 presents the evolution of type of faculty position over the course of each program year. Regardless of year, a majority of participants are interested in becoming tenure track faculty. Conversely, there is limited interest in pursuing non-tenure track positions. With the 2016-2017 program, we see more of an 
increase in pursuing TTRO positions over the first half of the program; there is a nominal increase in interest for TTTO positions during the same period. The dominance of research-related positions has reinforced our decision to expand expand research preparation and include a Job Talk module.

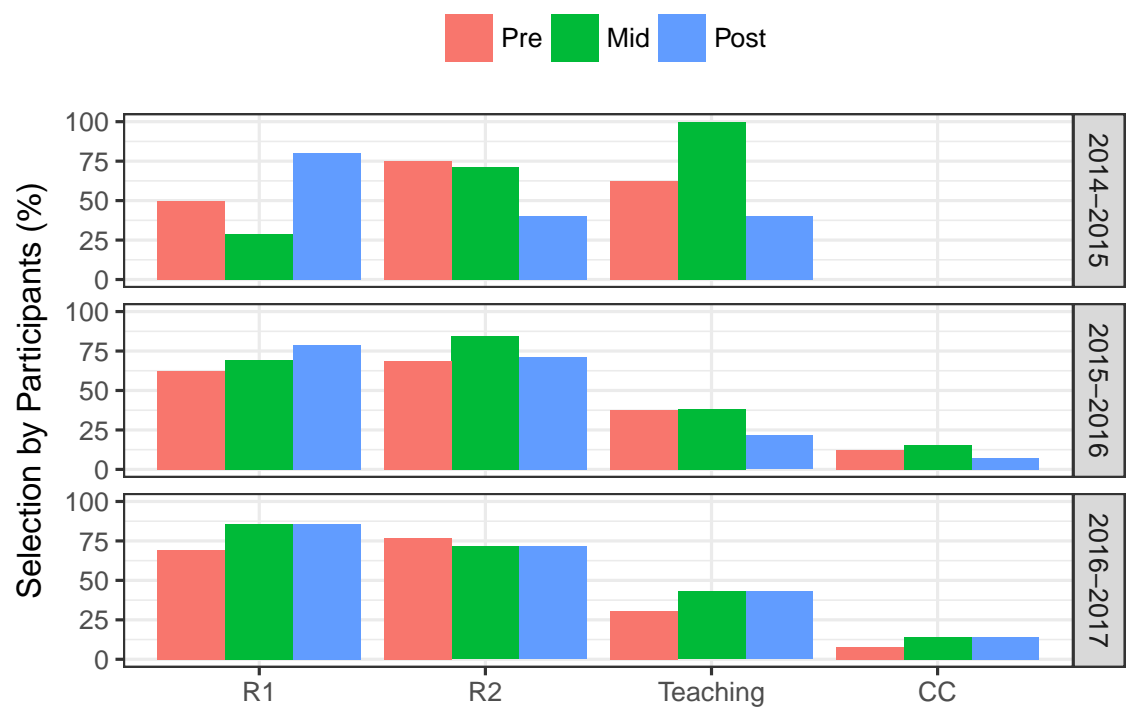

Figure 3: Types of institutions of interest by participants where $R 1$ refers to a research intensive institution, $R 2$ refers to Research and Teaching Balanced, Teaching refers to Teaching Intensive Institutions, and $C C$ refers to community colleges. Each row of the figure corresponds to a year of the program.

Figure 3 shows how the type of institution varied for each of the cohorts. In all years, most participants expressed an interest in obtaining a position at a research intensive institution. Notably, most participants would like to continue conducting research at either an R1 or R2-type school. The high level of interest in those settings may be due to the program occurring at an R1 institution. Surprisingly, there has been limited interest in pursuing opportunities at community colleges. The sustained participant interest in R1 and R2 institutions supports our decision to emphasize communicating one's current and future research interests in their faculty application materials.

Figure 4 incorporates the current program's participants feelings of preparation to allow comparison to previous cohorts. Compared to prior program years, the 2016-2017 cohort initially demonstrated lower 25th and 75th quartiles of preparedness. In other words, a majority of participants in the 2016-2017 group felt less initially prepared than participants at the same stage in prior years. However, and by mid-program, the mean level of preparation was comparable to the 2015-2016 program and better than the 2014-2015 program. While we are pleased with the increase in feelings of preparedness, these gains could be offset by not yet having tackled drafting the research statement unlike previous years.

Figure 5 also includes preliminary familiarity results from the 2016-2017 program year. Similar to the observed lower level of preparation by the current cohort, we have noticed the lowest mean level of familiarity with the faculty application process to date. The lack of familiarity is somewhat surprising due to the presence of two postdoctoral fellows in the cohort. Furthermore, current participants also demonstrate the largest gains by the midpoint of the program compared to the first two years. If we continued to rely on the periodic assessments, the nuances behind such significant increases would be lost. 


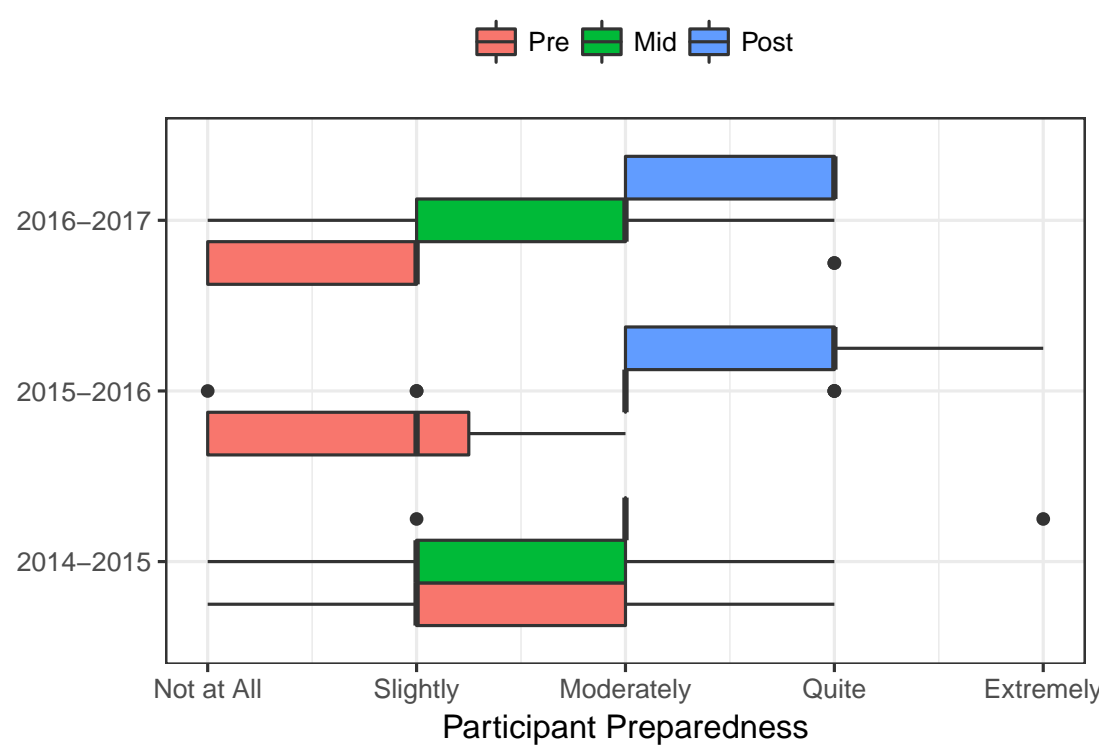

Figure 4: Participant impression of preparedness as a function of program year and timing of the survey. Participants across all program years indicate feeling "slightly" prepared at the start of the program. Both the 2015-2016 and 2016-2017 cohorts show improvement in preparedness from "slightly" to "moderately" by the midpoint of the program.

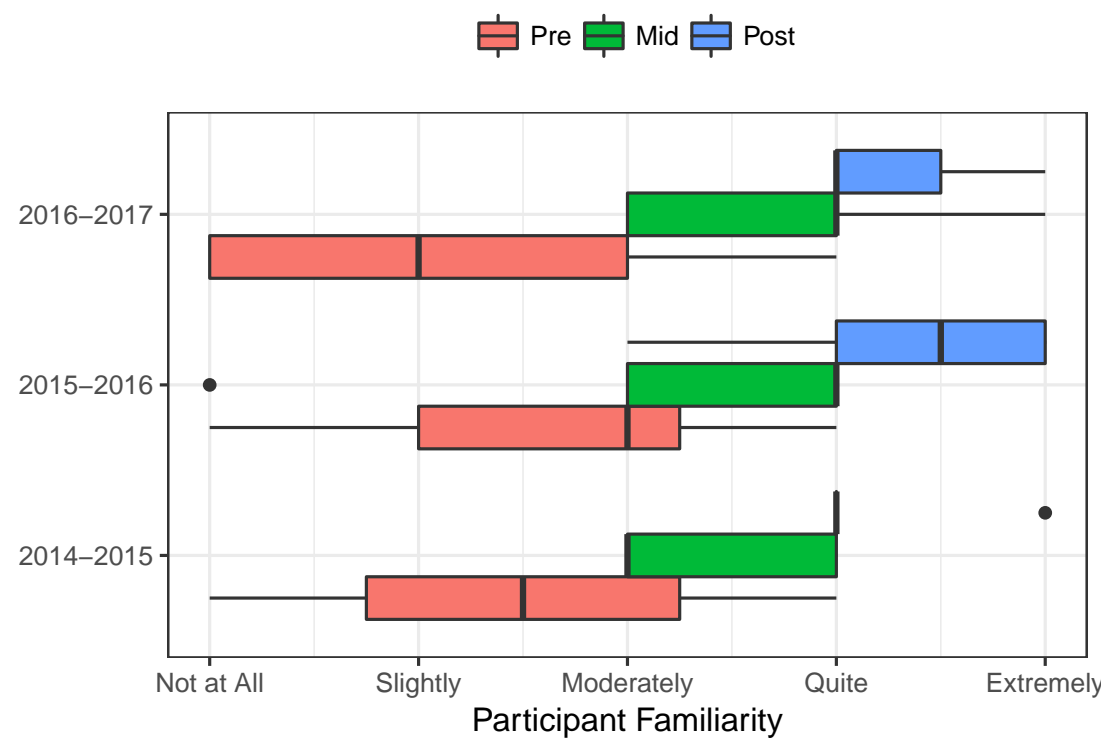

Figure 5: Participant impressions of familiarity with the faculty application process. Initial surveys of participants each program year indicate average feelings of "not at all prepared". While the 2014-2015 program sees a negligible change in preparedness, the 2015-2016 and 2016-2017 programs note gains in preparedness by mid-program.

\section{Most important and challenging application components}

Figure 6 identifies how participants rated the most important components of the faculty application packet. Cover letters were seen as consistently important in the first two years. However, no participants in the 
2016-2017 cohort deemed cover letters as an important component of the application packet. Similarly, no one felt the teaching statement was important at the time of the mid-program survey. We were surprised by this decline, because participants had attended a seminar and two peer review sessions on this topic. On the other hand, this cohort notes increased importance of the CV and research statements as they progressed through the first semester of the program.

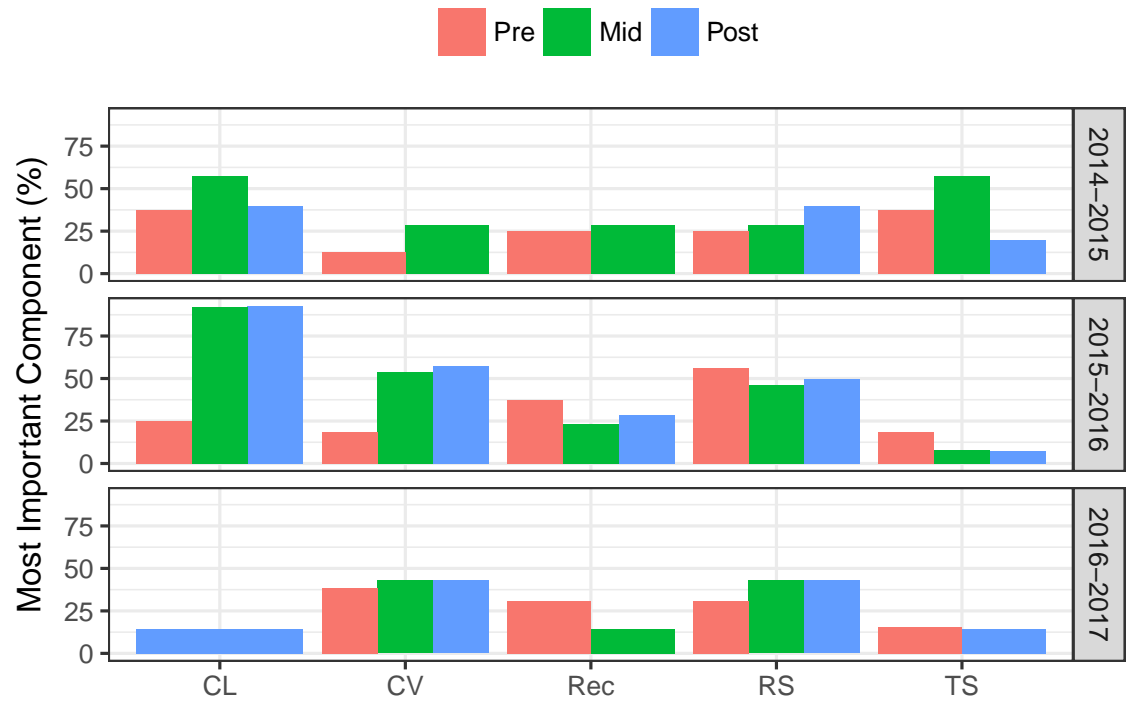

Figure 6: Selection of the most important components of the faculty application packet by program year. The cover letter viewed as extremely important through the 2014-2015 and 2015-2016 cohorts but was not considered extremely important by current program participants.

Figure 7 presents the components of the faculty application packet rated as most challenging by participants. The research statement is seen as the most challenging component by participants regardless of cohort. In Years 1 and 2, we observed a modest increase in preparing this component by the mid-point of the program. Pre- and mid-survey data for Year 3 participants demonstrates a similar trend. The continuous demonstration of difficulty in preparing the research statement was a motivating factor to expand the program to have a dedicated research statement section (see weeks 15-28 in Figure 1) in Year 3. The additional weeks allows us to further break down the research statement by offering additional two peer review opportunities for participants. We thought that these extra sessions would help de-mystify the research statement. However, and by the end of the second semester, a super majority of participants rated the research statement as the most challenging. One participant noted that their "research statement is extremely underdeveloped. I could have used more time to prepare/more initial lectures." The exhibited sentiment was echoed in another participant who felt that they still lacked a research idea, even after attending every session during the spring semester.

Figure 7 also shows an increasing trend in the number or participants in the 2016-2017 cohort who felt most challenged by the research statement. We believe this trend can be attributed to greatly awareness by participants of what truly makes a competitive research statement, especially given that most participants remain committed to joining faculty at research intensive institutions. It should be noted that the 2016-2017 trend counters the general trend from the program's first two years. 


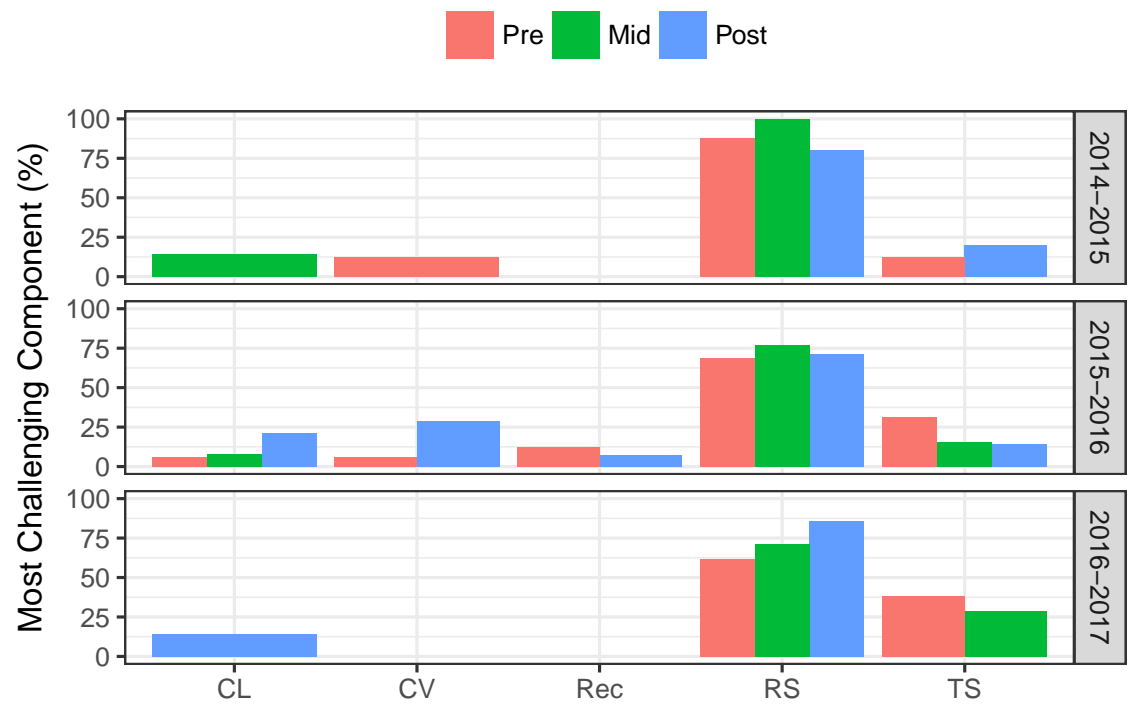

Figure 7: Selection of the most challenging components of the faculty application packet by program year. The research statement stands out in all program years as being exceptionally difficult for participants to prepare.

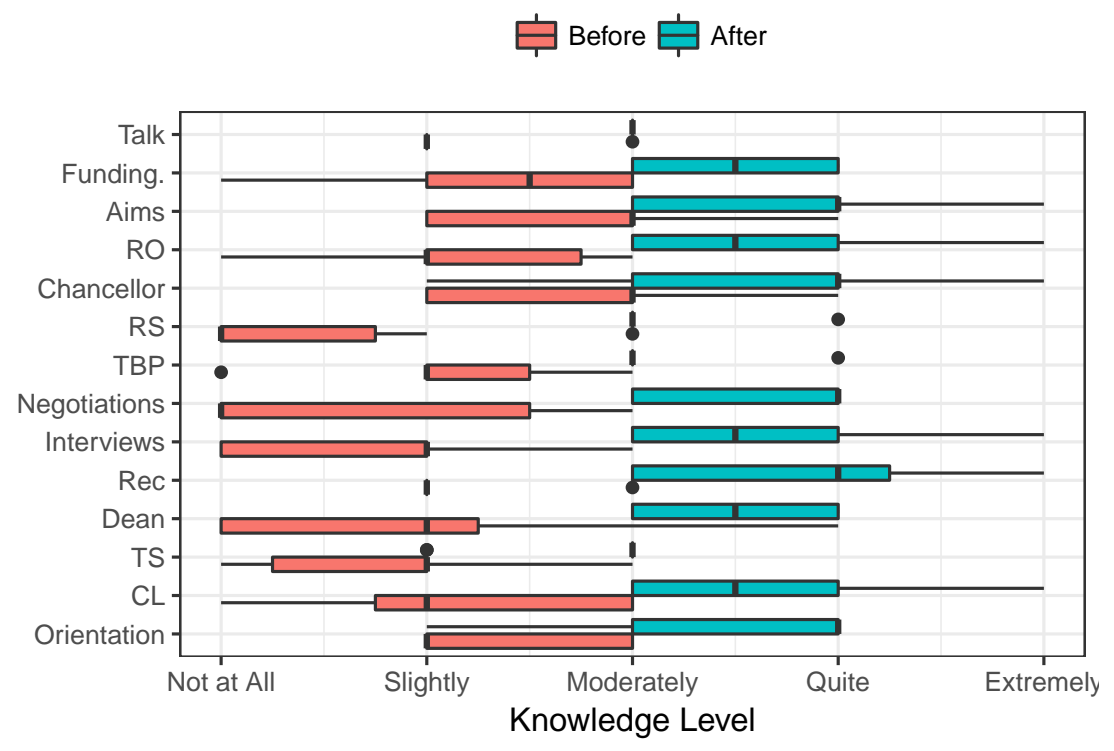

Figure 8: Changes in participant topical knowledge by attending panel and seminar sessions. Participants largely show increases in knowledge level by attending these sessions. $C L$ refers to cover letters, $T S$ refers to teaching statements, Dean refers to a discussion with the College of Engineering Dean, Rec refers to letters of recommendations, $T B P$ refers to the two-body problem, $R S$ refers to research statements, Chancellor refers to a discussion with the former campus Chancellor, $R O$ refers to research outlines, Aims refers to specific aims, and Talk refers to job talk.

\section{Enhancement of knowledge and preparedness}

The inclusion of the session surveys allowed us to capture for the first time specific changes in the cohort's topical knowledge and material preparation. Figure 8 shows changes in participant topical knowledge by 
attending the seminar and panel sessions. In all sessions, we find that participants improved their knowledge level from feeling either 'not at all' or 'slightly' knowledgeable about these topics to feeling either 'moderately' or 'quite' knowledgeable. These gains in knowledge could be attributed to faculty self-selection since they were contributing to sessions in which they themselves felt most knowledgeable.

As noted earlier, only $16 \%$ of the current cohort felt that the teaching statement was important at the beginning of the program versus $0 \%$ by the midpoint. Our initial thought was that the decline in perception of importance could be due to the teaching statement seminar. The in-session survey helped reveal to us a more complete picture of why this decline may have occurred. While Figure 8 shows an increase in participant knowledge level from slightly to moderately knowledgeable about teaching statements, it conflicts with trend shown in Figure 6. The teaching session is an example of how increased evaluation can uncover new insights into programming efficacy. In particular, we were able to capture one participant noting how they "...got the feeling from two of the panelists that the teaching statement isn't important, which is sort of discouraging." We feel that that this information would have been lost if we only had the mid-program survey data.

Building on observed trends in familiarity (see Figure 5), the in-session surveys allow us to trace back those knowledge gains to have a more complete understanding. In particular, we thought that some of these gains could be attributed to a revamped initial session. This year's first session was an orientation that also included an overview of the job application process and how to search for academic positions. The program introduction led to an immediate increase from slightly knowledgeable to quite knowledgeable for understanding how to obtain a faculty position.

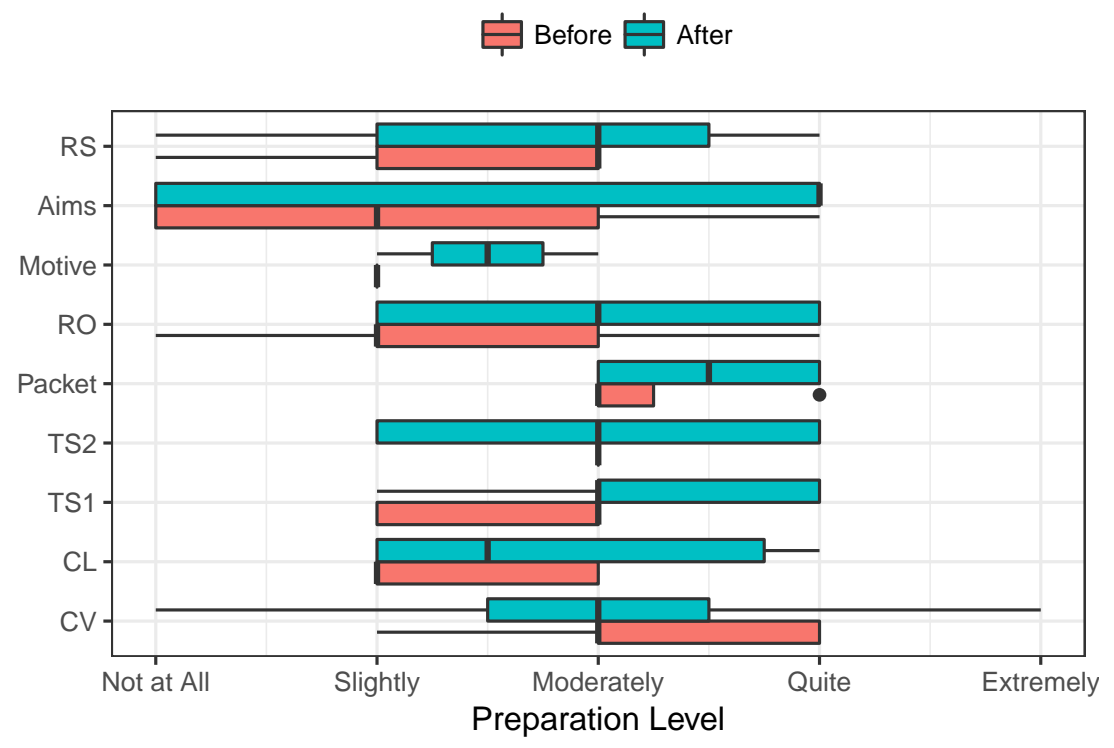

Figure 9: Changes in participant materials preparation by attending peer review sessions. Participants show increases in preparation level by attending these sessions. $C V$ refers to curriculum vita, $C L$ refers to cover letters, $T S 1$ refers to the first teaching statement review, $T S 2$ refers to the second teaching statement review, Packet refers to review of application materials completed thus far (includes CV, cover letter and teaching statement), $R O$ refers to research outline in preparation of the research statement, Motive revers to background and motivation statement, Aims refers to specific aims, and $R S$ refers to final draft of the research statement. 
Figure 9 presents changes in material preparation level as a result of attending the peer review sessions. Mean preparation levels increased over the course of the cover letter, fall packet (cover letter, CV, and teaching statement), and the research outline. Overall, there were no changes in mean preparation level for the $\mathrm{CV}$ and teaching statement drafts. Interestingly, the 25th - 75th quartile range (moderately to quite prepared) for the $\mathrm{CV}$ actually declined by attending the session. The in-session survey allowed us to qualitatively capture feedback by participants to understand this negative shift. One attendee remarked that they "realized [there was] so much to fix", whereas another participant wrote that they received "reviews that I've never gotten elsewhere". In general, we were pleased to see steady improvements in participant preparation level as they typically moved from slight and moderate preparation levels towards moderate and quite prepared levels. The additional intensity to focus on the research statement in the Spring ultimately did not result in participants feeling more prepared about this statement by the end of the semester.

\section{Incorporating feedback to improve program retention}

As stated, our program goal is to improve future female faculty's preparedness and familiarity with the academic job search. However, and as Figure 10 highlights, participant retention is an ongoing problem with our program. The addition of the weekly surveys also allows us to use the feedback to inform future programming decisions at the session level with the hopes of improving our overall retention.

With regard to seminars and panel sessions, we feel that the weekly feedback reinforced the selection of topics. On average, participants admitted that they were only slightly knowledgeable about most topics. Notably, sessions such as the research statement and negotiations highly topics where participants initially felt they had no knowledge at all. These are two difficult topics that may need additional coverage in future sessions. In fact, one participant noted how a practice session on negotiating would be helpful. Practice negotiations are presently on our list of potential peer review sessions for future program years.

We attribute the precipitous lack of peer review attendance on a couple of factors. First, and as organizers, we may not have been clear about program expectations for the 2016-2017 program year, especially with regard both program length and peer review. The current program represents a $114 \%(=8 / 7)$ and $67 \%$ $(=4 / 6)$ increase in the number of seminars and peer reviews versus the 2015-2016 program, respectively. While the program topics were posted on our website when people applied for admission, we still may not have been clear conveying how long was. The other factor for diminished attendance may be due to lack of cohort building. Our program is small and is annually limited to approximately 15 students. However, we demonstrated some degree of naiveté in thinking that the small size would automatically translate into a fully accountable cohort without any social scaffolding by us. Future programming could help alleviate this issue by adding in explicit cohort building activities such as social events at the beginning of the program.

\section{Improved tracking of attendance}

We largely view surveys as being reflective of the minimum number of attendees that are present. During the 2014-2015 and 2015-2016 program years, attendance was tracked by the number of surveys completed throughout the program. For 2016-2017 program, the introduction of the session surveys improved our attendance record keeping. Figure 10 presents the change in attendance over the course of the program. Attendance is normalized by the number of pre-surveys collected. The 2016-2017 weekly surveys allow us to quantitatively match fluctuations in attendance with corresponding programming. The largest drop-offs in attendance consistently coincide with peer review sessions. 


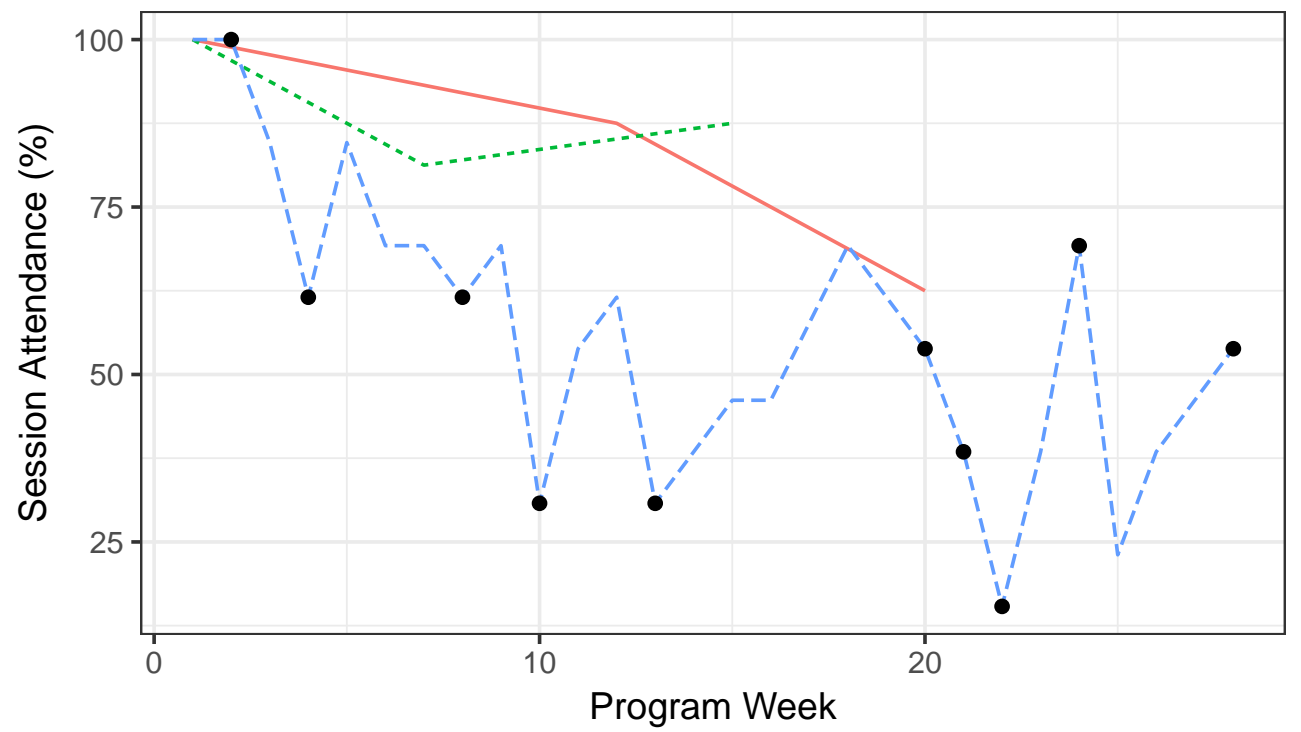

Figure 10: Participant retention during each program year. Attendance is based on the number of surveys completed at the time. Additional attendance data is available for the 2016-2017 year due to the inclusion of individual session surveys. Dots denote peer review sessions for the 2016-2017 cohort. Peer review session attendance is noticeably lower than panel/peer review sessions.

The lack of attendance has also not gone unnoticed by those who do attend the peer review. One participate noted that, " it would be nice if everyone who applied and was accepted to [the program] was dedicated to full packet development." As organizers, creating sense of belonging to a cohort to help improve accountability is one our priorities as we continue to review our current efforts. However, we do not believe that cohort building can be forced by transitioning the program from a voluntary experience to a formal class. In fact, choosing the latter is considered a "nuclear" option, and we will attempt more organic methods in the next program cycle to improve in this area.

\section{Faculty engagement}

We saw FIS response rates of 73.9\% (= 17/23) and 76.9\% (=10/13) for the Fall and Spring, respectively. We were not expecting to have such high response rates to our surveys. However, faculty often completed the FIS within minutes of receiving it. The advanced planning with faculty allowed us to have speaker calendars set prior to the semesters' commencement. This represents a dramatic difference compared to previous years in which we would have confirmation of speakers sometimes only one to two days prior to a session.

Additionally, the new approach to reaching out to faculty led decreases in reported time conflicts. For the 2016-2017 program year, only one faculty member expressed a conflict, and that was due to them being off campus for a sabbatical. By introducing the FIS, we were able to engage more faculty in our program. These increases also resulted in having a broader distribution of departments and research types (e.g., experimental, computational, etc.). 


\section{Conclusions}

We view intervention programs such the Illinois Female Engineers in Academia Training program as critical in helping combat the barriers to pursuing faculty careers by women and other under-represented groups in STEM. Program evaluation is essential in ensuring that we are imparting the best and most relevant information to our participants as they prepare materials and gain understanding of the the job search process. To that end, we have dramatically increased our program evaluation structure to incorporate same-session feedback. For the 2016-2017 program year, we are now able to identify in-session gains of participant topical knowledge as well as material preparation level. Without these surveys, we would be at a disadvantage in understanding how helpful individual program components are. Thus, this study highlights how incorporating more survey instruments can positively aid in targeted understanding of which components assist in the improving under-represented people in STEM's preparedness and familiarity to embark on the academic job search process. Furthermore, well-timed surveys can also be used to improve faculty participant rates and assist with matching faculty to topics that they are most knowledgeable in. Overall, we believe that the increase in evaluation helps keep our intervention current by assisting with refining our program.

\section{References}

[1] B.L. Yoder. Engineering by the numbers 2016, 2016. https://www.asee.org/papers-andpublications/publications/college-profiles/15EngineeringbytheNumbersPart1.pdf.

[2] M.T. Gibbons. Engineering by the numbers, 2009. https://www.asee.org/papers-andpublications/publications/college-profiles/15EngineeringbytheNumbersPart1.pdf.

[3] Helen Shen. Mind the gender gap. Nature, 495(7439):22, 2013.

[4] Rethink your gender attitudes. Nature Materials, 13(5):427, 2014.

[5] Corinne A Moss-Racusin, John F Dovidio, Victoria L Brescoll, Mark J Graham, and Jo Handelsman. Science facultys subtle gender biases favor male students. Proceedings of the National Academy of Sciences, 109(41):16474-16479, 2012.

[6] Sari M Van Anders. Why the academic pipeline leaks: Fewer men than women perceive barriers to becoming professors. Sex roles, 51(9-10):511-521, 2004.

[7] Diana Bilimoria, Simy Joy, and Xiangfen Liang. Breaking barriers and creating inclusiveness: Lessons of organizational transformation to advance women faculty in academic science and engineering. $\mathrm{Hu}$ man Resource Management, 47(3):423-441, 2008.

[8] E.R. Horstman, D.J. Mai, Y. Li, and R. Bhargava. A Program for Graduate Women in Engineering Pursuing Academic Careers. In 2015 Annual Conference \& Exposition. American Society for Engineering Education, 2015.

[9] Y. Li, D.J. Mai, E.R. Horstman, and R. Bhargava. Preparing female engineering doctoral students for the academic job market throgh a training program inspired by peer review. In 2015 Annual Conference \& Exposition. American Society for Engineering Education, 2015. 